\title{
Perfil epidemiológico e avaliação do risco de queda em idosos usuários da Estratégia
}

\section{Saúde da Família}

\author{
Epidemiological profile and risk assessment of falls in elderly users of the Family Health Strategy \\ Perfil epidemiológico y evaluación del riesgo de caídas em usuários mayores de la Estrategia Salud \\ de la Familia
}

Recebido: 06/09/2021 | Revisado: 15/09/2021 |Aceito: 18/09/2021 | Publicado: 19/09/2021

Lílian Karine Machado de Souza

ORCID: https://orcid.org/0000-0002-3809-7667 Universidade de Pernambuco, Brasil E-mail: lilian.souza@upe.br

Vitor Matheus Alves Pereira ORCID: https://orcid.org/0000-0001-8936-2144 Universidade de Pernambuco, Brasil E-mail: vitor.matheus@upe.br

Aron Nogueira Aquino

ORCID: https://orcid.org/0000-0003-3834-6309 Universidade de Pernambuco, Brasil E-mail: aron.aquino@upe.br

Maísa Maciel de Almeida

ORCID: https://orcid.org/0000-0001-5321-2769 Universidade de Pernambuco, Brasil

E-mail:maisa.maciel@upe.br

Joab Lins Serafim

ORCID: https://orcid.org/0000-0003-3699-2454 Universidade de Pernambuco, Brasil E-mail: joab.serafim@upe.br

Pauliana Valeria Machado Galvão ORCID: https://orcid.org/0000-0002-4418-218X Universidade de Pernambuco, Brasil pauliana.galvao@upe.br

Daniela de Araújo Viana Marques ORCID: https://orcid.org/0000-0002-2380-7910 Universidade de Pernambuco, Brasil E- mail: daniela.viana@upe.br

\begin{abstract}
Resumo
O presente estudo tem como objetivo identificar o perfil epidemiológico e avaliar a ocorrência de tonturas e risco de queda em idosos do município de Serra Talhada, Pernambuco, Brasil. Para tanto, foi realizado um estudo epidemiológico quantitativo, descritivo e transversal realizado com idosos com queixa de tontura e usuários da rede de Atenção Básica de Serra Talhada, de agosto de 2017 a agosto de 2018. Utilizou-se questionários acerca do perfil epidemiológico, história médica pregressa e ocorrência de tonturas. O risco de quedas foi avaliado pelo teste Timed Up and Go (TUG) com regressão logística da variável dicotomizada (presença ou ausência de dependência). Foram avaliados 101 indivíduos, com predominância para o sexo feminino, pardos, com ensino fundamental I incompleto, casados, aposentados e sedentários. A idade média foi de 71,1. As principais comorbidades foram Hipertensão Arterial Sistêmica, deficiências visuais e artrite. A média do TUG foi de 15,9 segundos, não havendo relação estatística significativa com o sexo, ocorrência de tonturas, atividade física ou autopercepção de saúde. Houve relação direta entre a dependência e a idade e a presença de diabetes mellitus e inversa com a presença de HAS. Constatou-se, através da análise do perfil epidemiológico e da identificação das variáveis que melhor contribuem para a ocorrência probabilística de dependência segundo os critérios do TUG, infere-se fatores de risco associados à queda, permitindo uma abordagem rápida e efetiva que poderá diminuir a sua ocorrência.
\end{abstract}

Palavras-chave: Envelhecimento; Equilíbrio postural; Tontura.

\section{Abstract}

This study aims to identify the epidemiological profile and assess the occurrence of dizziness and the risk of falling in elderly people in the municipality of Serra Talhada, Pernambuco, Brazil. Therefore, a quantitative, descriptive and cross-sectional epidemiological study was carried out with elderly people complaining of dizziness and users of the Primary Care network of Serra Talhada, from August 2017 to August 2018. Questionnaires were used on the 
epidemiological profile, history medical history and dizziness. The risk of falls was assessed using the Timed Up and Go (TUG) test with logistic regression of the dichotomized variable (presence or absence of dependence). 101 individuals were evaluated, predominantly female, brown, with incomplete primary education, married, retired and sedentary. The average age was 71.1. The main comorbidities were Systemic Arterial Hypertension, visual impairment and arthritis. The TUG mean was 15.9 seconds, with no statistically significant relationship with gender, occurrence of dizziness, physical activity or self-perceived health. There was a direct relationship between dependence and age and the presence of diabetes mellitus, and an inverse relationship with the presence of SAH. It was found, through the analysis of the epidemiological profile and the identification of the variables that best contribute to the probabilistic occurrence of dependence according to the TUG criteria, inferring risk factors associated with the fall, allowing a quick and effective approach that could reduce the its occurrence.

Keywords: Aging; Postural balance; Dizziness.

\section{Resumen}

Este estudio tiene como objetivo identificar el perfil epidemiológico y evaluar la ocurrencia de mareos y el riesgo de caídas en personas mayores en el municipio de Serra Talhada, Pernambuco, Brasil. Por lo tanto, se realizó un estudio epidemiológico cuantitativo, descriptivo y transversal con ancianos con queja de mareos y usuarios de la red de Atención Primaria de Serra Talhada, de agosto de 2017 a agosto de 2018. Se utilizaron cuestionarios sobre el perfil epidemiológico, antecedentes médicos y aparición de mareos. El riesgo de caídas se evaluó mediante la prueba Timed Up and Go (TUG) con regresión logística de la variable dicotomizada (presencia o ausencia de dependencia). Se evaluaron 101 individuos, predominantemente mujeres, morenos, con educación primaria incompleta, casados, jubilados y sedentarios. La edad media fue de 71,1 años. Las principales comorbilidades fueron hipertensión arterial sistémica, discapacidad visual y artritis. La media de TUG fue de 15,9 segundos, sin relación estadísticamente significativa con el sexo, aparición de mareos, actividad física o salud autopercibida. Existió una relación directa entre la dependencia y la edad y la presencia de diabetes mellitus, y una relación inversa con la presencia de HSA. Se encontró, a través del análisis del perfil epidemiológico y la identificación de las variables que mejor contribuyen a la ocurrencia probabilística de dependencia según los criterios TUG, inferiendo factores de riesgo asociados a la caída, permitiendo un abordaje rápido y efectivo que podría reducir la su ocurrencia.

Palabras clave: Envejecimiento; Equilibrio postural; Mareo.

\section{Introdução}

Em 2015, os idosos representavam 12\% da população mundial e a previsão é de que este quantitativo duplique até 2050 e triplique em 2100 (Suzman, et al., 2015). Esta maior longevidade pode ser considerada um sucesso para a humanidade, mas tem diversas implicações na vida dos próprios idosos e na sociedade, sobretudo no que tange à saúde (Mari, et al., 2016; Tavares, et al., 2017), visto que o processo de envelhecimento tem sido frequentemente acompanhado por problemas de saúde físicos e mentais provocados muitas vezes pela ocorrência prévia de doenças crônicas e quedas (Tavares, et al., 2017).

As alterações ocasionadas pelo processo do envelhecimento, apesar de previsíveis e progressivas, não são uniformes entre as pessoas. Fatores genéticos, estilo de vida e exposições ambientais interferem na variação da longevidade (Oliveira, et al., 2015). Essas modificações associadas ao envelhecimento afetam todos os sistemas sensoriais do corpo e causam degeneração das estruturas anatômicas do sistema vestibular, responsáveis pelo equilíbrio. Os distúrbios resultantes do déficit no equilíbrio corporal na velhice podem ser expressos através de sintomas subjetivos (como a tontura) e objetivos (como distúrbios de marcha e tendência a cair) (Zalewski, 2015).

O equilíbrio é o processo pelo qual o Sistema Nervoso Central (SNC) provoca os padrões de atividade muscular necessários para coordenar a ação entre o centro de massa (o peso corporal) e a base de suporte (as articulações, sobretudo joelhos e tornozelos). Percebe-se como uma função multissensorial, por envolver sistemas sensoriais, motores, o proprioceptivo e o vestibular (Bouccara, et al., 2018). Dessa forma, vários aspectos contribuem para a perda do equilíbrio, seja ela total ou parcial, tais como alterações no sistema vestibular, diminuição da contração muscular, diminuição da coordenação motora, alterações visuais, alteração da propriocepção, alterações cognitivas e diminuição da sensibilidade tátil (Schlick, et al., 2016).

A queixa de tonturas é a mais comum relatada pelos idosos com 75 anos ou mais, interferindo na saúde e na vida social de maneira severa e possuindo relação direta com diversas condições indesejadas, como o isolamento, a depressão e a 
redução da autonomia (Ciorba, et al., 2016). Cerca de 30\% das pessoas com 70 anos ou mais queixam-se de prejuízo às atividades diárias devido à tontura. Por estas razões, a tontura tem impacto direto na qualidade de vida da pessoa de mais idade (Jahn, 2019).

Independente da causa, a instabilidade postural conduz ao medo de cair, que é um importante fator preditor e precipitante de quedas. Outros preditores de risco de queda são mobilidade reduzida, atividade física pobre, severidade da tontura e fraqueza muscular. Identificar perfis de risco para quedas é mandatório na clínica, visto que são causas importantes de morbidade e mortalidade entre os idosos e lideram entre as causas de fraturas fatais e não fatais. Queda também é a maior causa de morte acidental nessa faixa etária, além de ser custosa aos serviços de saúde (Cuevas-Trisan, 2019; Laurence; Michel, 2017; Vanspauwen, 2018)

Sendo assim, diante do envelhecimento populacional brasileiro, estima-se o crescimento dos quadros de tontura, das quedas e suas intercorrências, tais como fraturas de quadril e/ou fêmur, que podem levar a diminuição da mobilidade e independência e, em casos mais graves, ao óbito. A identificação do perfil dos idosos com essa queixa e a avaliação da mobilidade e do equilíbrio permitirão um melhor planejamento de políticas públicas para este grupo etário. Nesse sentido, os objetivos deste estudo foram descrever o perfil epidemiológico e avaliar o risco de quedas em idosos com déficit de equilíbrio usuários da Estratégia Saúde da Família (ESF) no município de Serra Talhada, Pernambuco.

\section{Metodologia}

Trata-se de um estudo epidemiológico descritivo, transversal e com abordagem quantitativa, tendo em vista o enfoque na determinação de tendências e indicadores, bem como interesse no nas características do grupo, ou seja, no coletivo (De Freitas Mussi, et al., 2019). Para tanto, foi realizada uma pesquisa de campo com idosos com queixa de tontura e usuários da rede de Atenção Básica do município de Serra Talhada - PE no período de janeiro de 2018 a dezembro de 2018. Este estudo foi realizado após aprovação do projeto pelo Comitê de Ética em Pesquisa da Faculdade de Ciências Médicas da Universidade de Pernambuco (UPE) (CAAE 74873717.0.0000.5207 e parecer de aprovação nº 2.414.896/2017 em 04 de dezembro de 2017). Todos os participantes procederam à assinatura de Termo de Consentimento Livre e Esclarecido (TCLE) em duas vias antes da realização de quaisquer procedimentos da pesquisa, assumindo-se o compromisso de preservação da privacidade dos pacientes.

O critério de inclusão foi a queixa declarada de tonturas em idosos usuários da Atenção Básica, lúcidos, responsivos e na faixa etária estipulada. Os critérios de exclusão foram a recusa em participar do estudo, ausência de queixa de tontura e incapacidade de responder às questões. Ou seja, foi questionada a frequência com que se dava a queixa de tontura: 'Sempre', 'Às Vezes', 'Raramente', 'Nunca' ou 'Não Sei Responder'. Os que responderam 'Nunca' ou 'Não Sei Responder' preencheram critério de exclusão e não prosseguiram no estudo. Os demais completaram o questionário sociodemográfico e foram submetidos ao teste de aptidão física Timed Up and Go (TUG).

O questionário sociodemográfico continha informações referentes a: sexo, etnia, estado civil, escolaridade e ocupação. Foi questionada a realização eventual de alguma atividade física e a existência de doenças crônicas ou comorbidades: Hipertensão Arterial Sistêmica (HAS), Diabetes Mellitus (DM), doenças pulmonares, doenças cardíacas, deficiências visuais, deficiências auditivas, câncer, doença de Parkinson, Acidente Vascular Encefálico (AVE) prévio, fratura prévia, artrite e labirintite. No que se refere à autopercepção da qualidade de vida, foi questionado ao idoso se ele se considerava saudável, sendo possíveis as seguintes respostas: "Sim", "Não", "Mais ou menos" ou "Não sei responder".

O TUG, instrumento validado que avalia equilíbrio, mobilidade, desempenho muscular e risco de quedas, foi aplicado segundo o preconizado pelos autores Podsiadlo (1991) e Dutra, Cabral e Carvalho (2016). No TUG, o idoso é encorajado a desempenhar a marcha sem auxílio a partir de um ponto inicial (cadeira com encosto traseiro), levantando-se e caminhando 
três metros em linha reta, para então girar 180 graus em seu próprio eixo e retornar à posição inicial. O tempo decorrido nesse trajeto - desde quando o comando para se levantar é dado até o momento em que o idoso é restituído à posição inicial - é anotado e classificado em: $\leq 10$ segundos - idoso independente, sem alterações; $11-20$ segundos - idoso independente em transferências básicas, baixo risco de quedas; $\geq 20$ segundos - idoso dependente em vários aspectos das atividades cotidianas, alto risco de quedas (Mathias; Nayak; Isaacs, 1986; Bischoff, et al., 2003). Os participantes que não desejavam ou não foram aptos à realização do TUG não pontuaram.

Os dados foram analisados no programa estatístico R versão 3.6.2. O TUG foi mostrado como mediana e intervalo interquartílico, visto que as variáveis numéricas (TUG e idade) não seguiam a distribuição normal. A idade para ser mais compreensível e comparável foi apresentada como média e desvio padrão. A análise descritiva das variáveis categóricas foi apresentada como valores absolutos e relativos (em porcentagem). Para comparação dos resultados médios nos testes de equilíbrio, foi utilizado o teste t de Mann-Whitney. O nível de significância adotado foi de 0,05 ( $\alpha=5 \%)$.

Para a análise de regressão logística, foram considerados apenas os participantes que executaram o TUG e a variável foi dicotomizada como: Presença de dependência (tempo $>10$ segundos) e ausência de dependência (tempo $\leq 10$ segundos). Em síntese, foi feita regressão múltipla com uma variável de saída categórica e dicotômica e variáveis previsoras contínuas ou categóricas. Permitiu-se, assim, prever a qual de duas categoriais é mais provável que uma pessoa pertença, dadas certas informações (Field, 2011), ou seja, foi possível prever a probabilidade de uma observação estar relacionada a um evento de interesse - neste caso, presença de dependência - em relação a um grupo relacionado à ausência deste evento (Battisti; Smolski, 2020) e estimar os coeficientes através da máxima verossimilhança (Dobson, 2002).

Primeiramente, várias regressões logísticas simples foram realizadas para identificar as possíveis variáveis do modelo completo. As características sociodemográficas e a presença de comorbidades foram dicotomizadas para facilitar a modelagem. Além destas, a idade foi acrescida como variável contínua e a realização de atividades físicas também foi incluída. Os modelos de regressão logística simples que apresentaram $p$-valor $<0,20$ - revelando significância estatística de $20 \%$ - foram incluídos à regressão logística múltipla, a saber: Idade $(p=0,13)$, Atividade Física $(p=0,15)$, Tontura $(p=0,20)$, HAS $(p=$ $0,10), \operatorname{DM}(p=0,15)$, Deficiência visual $(p=0,142)$. A variável 'sexo' também foi incluída devido a achados na literatura.

Com estas variáveis, a regressão logística múltipla foi executada pelo método automático Stepwise empregado no sentido both. Este método, por sua vez, utiliza o Critério de Informação de Akaike (Akaike Information Criterion ou AIC) na combinação das variáveis dos diversos modelos simulados para selecionar o modelo mais ajustado. Quanto menor o AIC, melhor o ajuste do modelo (Dobson, 2002; Battisti; Smolski, 2020). Após estimar o melhor modelo, as razões de chance (Odds ratio ou OR) foram estimadas com intervalos de confiança de 95\%. A multicolinearidade foi testada através do teste de Fator de Inflação de Variância (VIF) para averiguar se alguma das variáveis incluídas atrapalhava a interpretação de outra, e os valores encontrados devem resultar em valores maiores que 10 para representar baixo problema de multicolinearidade (Rawlings; Pantula; Dickey, 1998). Todas as variáveis incluídas tiveram VIF acima de 10. O teste de Hosmer e Lemeshow foi realizado para demonstrar a qualidade de ajuste do modelo. O Pseudo $R^{2}$ foi calculado para evidenciar o ajuste geral do modelo proposto, que representa a melhoria proporcional na função log-verossimilhança em função dos termos do modelo de interesse comparado ao modelo mínimo (Dobson, 2002). Sua interpretação é similar à regressão múltipla (Battisti; Smolski, 2020).

\section{Resultados}

Foram testados 101 indivíduos, com predominância na amostra para o sexo feminino, pardos, com ensino fundamental I incompleto, casados, aposentados e sedentários (não fazem atividade física regular). A idade média foi de 71,1 $( \pm 7,8)$ (Tabela 1). As principais comorbidades referidas foram HAS, deficiências visuais e artrite (Tabela 2). A tontura foi referida com a maior frequência de "às vezes” (Tabela 3 ). 
Tabela 1: Características sociodemográficas $(n=101)$.

\begin{tabular}{cc}
\hline Masculino & $20(19,8)$ \\
Feminino & $81(80,2)$ \\
Idade (Média \pm desvio padrão) & $71,1 \pm 7,8$ \\
Branco & $45(44,6)$ \\
Pardo & $47(46,5)$ \\
Preto & $9(8,9)$ \\
Analfabeto & $23(22,8)$ \\
Ensino Fundamental I Incompleto & $43(42,6)$ \\
Ensino Fundamental I Completo & $15(14,9)$ \\
Ensino Fundamental II Incompleto & $6(5,9)$ \\
Ensino Fundamental II Completo & $5(4,9)$ \\
Ensino Médio Incompleto & $2(2,0)$ \\
Ensino Médio Completo & $6(5,9)$ \\
Superior Completo & $1(1,0)$ \\
Solteiro & $18(17,8)$ \\
Casado & $36(35,6)$ \\
União estável & $1(1,0)$ \\
Divorciado & $12(11,9)$ \\
Viúvo & $34(33,7)$ \\
Do lar & $19(18,8)$ \\
Aposentado & $76(75,3)$ \\
Agricultor & $5(4,9)$ \\
Outra ocupação & $5(1,0)$ \\
Faz atividade física & $43(42,6)$ \\
Não faz atividade física & $46(45,5)$ \\
Àsez faz atividade física & $12(11,9)$ \\
\hline
\end{tabular}

Fonte: Autores (2021).

Tabela 2: Dados clínicos dos idosos $(\mathrm{N}=101)$.

\begin{tabular}{cc}
\hline Hipertensão & $78(77,2 \%)$ \\
Deficiência Visual & $71(70,3 \%)$ \\
Artrite & $52(51,5 \%)$ \\
Labirintite & $37(36,6 \%)$ \\
Deficiência Auditiva & $28(27,7 \%)$ \\
Doenças cardíacas & $27(26,7 \%)$ \\
Diabetes & $24(23,8 \%)$ \\
Fratura & $18(17,8 \%)$ \\
Doenças pulmonares & $16(15,8 \%)$ \\
Acidente Vascular Encefálico & $8(7,9 \%)$ \\
Câncer & $3(3,0 \%)$ \\
Doença de Parkinson & $1(1,0 \%)$ \\
\hline
\end{tabular}

Fonte: Autores (2021). 
Tabela 3: Relato de tontura dos idosos $(\mathrm{n}=101)$

\begin{tabular}{ll}
\hline Relato de tontura & \\
Sempre & $22(22,8 \%)$ \\
Às vezes & $64(63,4 \%)$ \\
Raramente & $15(14,8 \%)$ \\
\hline
\end{tabular}

Fonte: Autores (2021).

Quanto ao teste TUG, 85,2\% dos participantes realizaram o teste. O tempo médio foi de $15,9 \mathrm{~s}( \pm 7,4)$, sendo mais frequente a realização entre 10 e 19 segundos. O teste de Mann-Whitney afirma que o desempenho do teste TUG por sexo não difere entre si, revelando que não houve significância estatística entre sexo e tempo utilizado na realização do teste. O mesmo se observa para a ocorrência de tonturas, realização de atividade física e autopercepção de saúde dos idosos (Tabela 4).

Tabela 4: Realização do teste Timed up and go.

\begin{tabular}{lc}
\hline Realização do teste $(\mathbf{n}=\mathbf{1 0 1})$ & $86(85,2 \%)$ \\
Sim & $5(4,9 \%)$ \\
Não conseguiu & $10(9,9 \%)$ \\
Recusou-se & \\
Tempo para realização do teste $(\mathbf{n}=\mathbf{8 6})$ & $7(8,1 \%)$ \\
Até 10 segundos & $63(73,3 \%)$ \\
Entre 10 e 19 segundos & $12(13,9 \%)$ \\
Entre 20 e 29 segundos & $4(4,7 \%)$ \\
Mais que 30 segundos & $15,9 \pm 7,4$ \\
Tempo médio \pm Desvio padrão* & $(\mathrm{p}=0,72)$ \\
Mediana e IQR por sexo** & $12,8 \mathrm{~s} \pm 6.76$ \\
Masculino & $14,6 \pm 4,98$ \\
Feminino & $(\mathrm{p}=0,25)$ \\
Mediana e IQR por realização de atividade & \\
física & $13,9 \pm 5,3$ \\
Sim & $15,0 \pm 8,6$ \\
Não & $(\mathrm{p}=0,52)$ \\
Mediana e IQR por autopercepção de saúde & $14,0 \pm 5,71$ \\
Sim & $15,6 \pm 3,7$ \\
Não & $(\mathrm{p}=0,24)$ \\
Mediana e IQR por frequência de Tontura & $14,6 \pm 5,0$ \\
Frequentemente & $12,8 \pm 5,7$ \\
Raramente &
\end{tabular}

Fonte: Autores (2021).

A Tabela 5 demonstra o OR estimado para as variáveis independentes (idade, HAS e DM) do modelo de presença de dependência segundo o teste TUG e evidencia que quanto maior a idade, a chance de aumento da dependência aumenta $8 \%$ a cada ano, a presença de DM aumenta em 10,29 vezes a chance de dependência, enquanto a presença de HAS aparenta reduzir a chance da presença de dependência em $90 \%$. O modelo proposto foi significativo, com boa qualidade de ajuste de modelo, a multicolinearidade foi testada e evidenciou uma possibilidade baixa entre as variáveis presentes no modelo proposto. O poder explicativo do modelo expresso pelo pseudo $R^{2}$ foi de 0,097 , o que evidencia que outras variáveis precisam ser estudadas para melhor explicar o agravo estudado.

Tabela 5: Odds Ratio estimado para as variáveis do modelo de presença de 


\begin{tabular}{ccccc}
\multicolumn{2}{l}{ dependência segundo o TUGT } & & & \\
\hline \multirow{2}{*}{ Idade } & OR & IC95\% & $p$ & VIF \\
& 1,08 & 0,$98 ; 1,28$ & 0,21 & 19, \\
Hipertensão & 0,10 & 0,$004 ; 0,94$ & 0,06 & 19, \\
& & & & 13 \\
Diabetes & 10,29 & 1,$18 ; 219,71$ & 0,05 & 20, \\
& & & & 60 \\
\hline
\end{tabular}

Fonte: Autores (2021).

\section{Discussão}

A ocorrência de quedas é um dos principais agravos que afetam a população idosa, sendo considerada um problema de Saúde Pública. É responsável pela perda da independência funcional, pela maioria das internações e cirurgias em idosos, causando elevados custos econômicos e sociais e sobrecarga dos serviços de saúde. As quedas representam a terceira causa mais comum de morte acidental no Brasil (Khow; Visvanathan, 2017; Abreu, et al., 2018) observando-se tendência de aumento das internações, mortalidade e letalidade por quedas em idosos entre 1998 e 2015 (Stolt, et al., 2020). Um estudo atual apontou um crescimento expressivo no número de internações por quedas em idosos no Brasil: em seis anos, aumentou de 2,58 para 41,37 por 10.000 habitantes, não sendo indicada a taxa de crescimento anual (Abreu, et al., 2018)

O perfil epidemiológico dos idosos incluídos no presente trabalho foi similar a outras pesquisas que buscaram avaliar o risco de quedas em população idosa usuária da ESF, com amostra predominantemente do sexo feminino, de idade média de 71,1 ( \pm 7,8), casados, sedentários e com acúmulo de comorbidades autorrelatadas (Santos, et al., 2015; Cruz, et al., 2012; Dantas, et al., 2012). Com esses resultados, objetivou-se demonstrar a importância das diferentes condições de saúde para a ocorrência de quedas, destacando a relevância da atuação da Atenção Básica na sua prevenção, especialmente em idosos com queixa de tontura, já que estes eventos são evitáveis e os profissionais de saúde podem ajudar na redução do risco das mesmas (Moreland; Kakara; Henry, 2020).

Nesta amostra estudada foi observado que, quanto maior a idade do idoso, menor o desempenho no TUG. Outros autores, analisando idosos e correlacionando idade e tempo gasto no TUG, evidenciaram associação semelhante (Rodrigues, et al., 2017; Pavanate, et al., 2018). Observou-se neste trabalho que o tempo gasto no teste tendeu a um incremento de $8 \%$, em média, para cada ano de idade, que pode ser explicado pelo fato de que o avanço da idade é um conhecido fator para piores resultados em testes de equilíbrio e mobilidade, contribuindo para o aumento do risco de queda (Ferreira, et al., 2019). Em contrapartida, estudo realizado com idosos brasileiros, no qual cerca de $30 \%$ possuíam queixa de tontura e $50 \%$ de desequilíbrio, e de grupo amostral de tamanho semelhante ao deste estudo, não foi constatado prejuízo no desempenho do TUG com o avanço da idade, o que foi justificado pelo tamanho do grupo e por cerca de $75 \%$ dos idosos possuírem 75 anos ou mais. Apesar disso, a idade foi associada a um maior número de quedas em indivíduos com queixa de desequilíbrio e queixa de tontura (Kurosawa; Shimazu; Yamamoto, 2020).

A idade é um fator de relevância, entendendo que o avançar progressivo dos anos também é capaz de reduzir a qualidade do equilíbrio, tanto estático quanto dinâmico, quando comparado a faixas etárias mais novas (Pavanate, et al., 2018). A prevalência de marcha anormal, que contribui com a chance de quedas, sofre um incremento com a idade e é maior nos idosos que vivem em instituições de longa permanência e em ambiente hospitalar de urgência (Vanspauwen, 2018), levando à reflexão de quanto o aumento progressivo de idosos no país e o proporcional surgimento de instituições de longa permanência, podem impactar, por exemplo, nos níveis de queda e comorbidades. 
A cidade em que o presente estudo foi desenvolvido - Serra Talhada, estado de Pernambuco - teve um aumento na expectativa de vida de 69,12 anos nos anos 2000 para 72,97 em 2010. O aumento da esperança de vida média para a população brasileira como um todo, no mesmo período, foi de 69,80 para 73,90. Vale ressaltar também o fato de o Índice de Desenvolvimento Humano (IDH) do município $(0,661)$ ser menor que o nacional $(0,759)$; o IDH agrega a longevidade da população no cálculo geral do índice, logo, menor longevidade implica menor IDH. Entretanto, a esperança de vida municipal se aproxima bastante da nacional, levando a crer que os demais componentes do IDH também são mais baixos que a média do país, de acordo com dados do Instituto Brasileiro de Geografia e Estatística [IBGE] (2010).

$\mathrm{O}$ fato de a expectativa de vida da população estudada ser semelhante à média nacional leva a crer que as taxas de déficit de equilíbrio em idosos sejam, também, semelhantes. Na presente amostra (média de idade de 71,1 $\pm 7,8$ ), a idade se relacionou diretamente com o declínio do equilíbrio, assim como ocorreu em outras pesquisas que se propuseram a estudar essa relação (Rodrigues, et al., 2017; Pavanate, et al., 2018). Vale lembrar que indivíduos muito fragilizados e incapazes de executar o TUG (possivelmente indivíduos de mais idade) preencheram critério de exclusão, logo, é possível que a média real de idade dos pacientes com déficit de equilíbrio seja ligeiramente maior.

O tempo gasto para realização do TUG não diferiu em função do sexo neste trabalho. Outros autores que se propuseram a estudar a relação entre essas duas variáveis também constataram esse achado. Em estudo realizado em FortalezaCE com população semelhante (idade até 79 anos $=57,8 \%$; sexo feminino predominante na amostra $=64,4 \%$ ), a variável sexo também não influenciou no tempo de execução do TUG ( $p=0$,691) (Chagas, et al., 2018). Outro estudo realizado em Patos $\mathrm{PB}$, a menos de $200 \mathrm{~km}$ do local de realização da presente pesquisa, também com população semelhante (média de idade $=71$ anos; sexo feminino predominante na amostra $=68.4 \%$ ), não encontrou diferença estatística entre os sexos no quesito tempo de execução do TUG ( $p=0.744$ ) (Rodrigues; Assef; Lima, 2019). Vale ressaltar a heterogeneidade dos estudos que determinaram a relação entre sexo e TUG. Em metanálise, o valor do TUG calculado para cada sexo foi significativamente heterogêneo entre os estudos $(p<0,01)$ (Long, et al., 2020). Dessa forma, apesar de o sexo não ser um preditor de risco ideal para déficit de equilíbrio e quedas na maioria dos estudos, deve-se levar em consideração a heterogeneidade dos resultados, e possivelmente seja prudente individualizar as populações. No presente estudo, no entanto, outras variáveis se demonstraram melhores na estratificação do risco em função do TUG.

Alterações visuais (National Academies of Sciences, Engineering, and Medicine, 2017), artrite (Vennu; Bindawas, 2014; Dore, et al., 2015) e HAS (Araújo, et al., 2017) são considerados fatores de risco para disfunção de marcha e equilíbrio e também estão associados à ocorrência de quedas. O risco de quedas parece ser diretamente proporcional ao número de fatores de risco que um indivíduo possui, de modo que aquele com maior probabilidade de cair pode ser precocemente detectado (Araújo, et al., 2017). Dentre as comorbidades mais referidas pelos idosos investigados nesta pesquisa destacam-se, em ordem de acometimento, HAS, deficiência visual e artrite, demonstrando que a presente amostra possui fatores de risco intrínsecos reconhecidos para quedas.

Quanto à realização do TUG, seu tempo de execução tem importância reconhecida na literatura. Por este teste, tempos superiores a 12 segundos caracterizam o idoso como em alto risco de dependência (Santos, et al., 2015; Browne; Nair, 2019). O TUG vem sendo estudado cada vez mais, na tentativa de avaliar a capacidade do teste como preditor de queda em idosos. Apesar de autores considerarem essa análise ainda limitada e influenciada por outras variáveis, estudo retrospectivo evidenciou associação significativa positiva entre o tempo de realização do teste e histórico de quedas em idoso. Além disso, o teste foi relatado como de sensibilidade considerável quando se trata de idosos com dependência funcional (Roshdibenam, et al., 2021). Sendo assim, o TUG foi sugerido como uma ferramenta clínica útil para aqueles idosos que têm alto risco de quedas, devendo ser sempre associado a outras variáveis, como uso de medicamentos, comorbidades e outros fatores sociodemográficos (Barry, et al., 2014; Kojima, et al., 2015) 
Neste estudo, o tempo médio de realização do TUG foi aproximado ao encontrado em estudos com populaçães semelhantes (Marchetti, et al., 2011; Roca, et al., 2014). Apesar disso, em uma metanálise foi calculado um tempo inferior, precisamente de 9,4 segundos, como o valor de tempo médio de realização deste teste após análise de 21 estudos que totalizaram 4395 casos (Bohannon, et al., 2006). É importante destacar que a referida metanálise incluiu vários estudos em indivíduos aparentemente normais ou grupos de controle normais, de tal forma que não houvesse interferência de patologias nos escores do TUG, objetivando, assim, propor bons valores de referência para este teste. Paralelamente, em estudo conduzido por Pondal e del Ser (2008) que incluiu 308 idosos domiciliados sem distúrbios da marcha ao exame neurológico, avaliados por um único neurologista com procedimento bem definido, determinou-se 10,2 segundos como pontuação de tempo médio no TUG. A partir disso, percebe-se que o tempo médio obtido no TUG nesta pesquisa é evidentemente maior que os observados nos estudos normativos em idosos saudáveis. As comorbidades apresentadas pelos participantes, em especial a tontura, poderiam justificar essas diferenças, já que exercem uma significativa influência no equilíbrio corporal. Desse modo, os resultados desta investigação coincidem com os estudos que afirmam que idosos com histórico de tontura e desequilíbrio têm maior risco de cair, visto que ao exibir um tempo maior no TUG, comparados aos valores normativos ou de referência, haveria uma maior predisposição à queda.

O aumento da longevidade populacional nos últimos anos, desencadeou um incremento na incidência de doenças crônicas, cuja prevalência aumenta com a idade tendo por exemplo DM e HAS (Ansai, et al., 2018). No que tange ao DM, evidenciou-se que os pacientes diabéticos desta amostra apresentaram chance de queda 10 vezes maior que os participantes não diabéticos. A correlação do risco de queda em pacientes diabéticos é tratada em diversos estudos recentes que utilizaram o TUG (Brown, et al., 2015; Waisman, 2017; Kang, et al.,2018), visto que a neuropatia diabética é sabidamente uma das causas mais comuns de comprometimento do equilíbrio em idosos. Alguns estudos normativos de referência (Brown, et al., 2015; Kang, et al.,2018), embora não tragam o valor específico do aumento do risco, encontraram através do teste TUG, o resultado de que pacientes com diabetes têm maior probabilidade de cair quando comparados aqueles que não possuem a doença. Já outros estudos (Hewston; Deshpande, 2016; Timar, et al., 2016), demonstraram que os pacientes diabéticos têm entre 5 e 6 vezes mais risco de cair em relação aos pacientes não diabéticos, dados inferiores ao encontrado neste trabalho.

O diagnóstico de DM tem se tornado cada vez mais frequente na área da otoneurologia em virtude da sua alta prevalência em pacientes com doenças labirínticas. A tontura, um dos sintomas mais recorrentes nesses pacientes, tem sido apontada como uma nova complicação do DM e um potencial fator de risco para quedas. A insulina e os níveis séricos de glicose exercem grande influência sobre as alterações vestibulares periféricas, de tal modo que, esses níveis são diretamente proporcionais à predisposição do paciente em desenvolver disfunções vestibulares (Silva, et al., 2019; Gioacchini, et al., 2018).

Apesar do cenário de alta prevalência de DM e da relação com alterações no equilíbrio ser bem estabelecida, poucos são os estudos que trazem informações sobre a associação entre DM e vertigem no contexto da Saúde Pública. A presente pesquisa ao reconhecer as doenças associadas ao déficit de equilíbrio, como a DM, contribui para a elaboração de intervenções preventivas eficazes e oferecimento de um manejo adequado dos pacientes, já que tal comorbidade pode originar e / ou exacerbar as alterações do déficit de equilíbrio (Silva, et al., 2019).

Revisões recentes na literatura (Sorond, et al., 2015; Coelho-Junior, et al., 2018; Ibrahim; Singh; Shahar, 2017) descreveram uma possível associação entre níveis pressóricos elevados, comprometimento da mobilidade/funcionalidade em idosos e baixo desempenho no TUG. Um dos mecanismos sugeridos para justificar a diminuição da funcionalidade muscular causada pela HAS seria o dano vascular constante nas artérias responsáveis pela irrigação de áreas cerebrais responsáveis pela mobilidade (Acar, et al., 2015). Apesar disso, neste estudo não houve associação entre HAS e baixa funcionalidade, visto que o grupo de pacientes hipertensos apresentou um desempenho significativamente melhor no TUG do que os normotensos. Estudo promovido por Coelho Júnior, et al., (2016) que utilizou o TUG como um dos testes para avaliações físicas e funcionais de 
idosas hipertensas e normotensas também concluiu que a hipertensão não se associou a baixa capacidade física e funcional. É válido ressaltar que tal estudo apenas incluiu pacientes do sexo feminino e excluiu àquelas com queixa de tontura. Além disso, é preciso pontuar que certamente o presente trabalho é o primeiro a analisar uma possível associação entre HAS e funcionalidade através do TUG em idosos com déficit de equilíbrio.

A HAS pode vir acompanhada de sintomas como taquicardia, turvação de visão, fraqueza; fatores que podem afetar o equilíbrio postural (Acar, et al., 2015). Além disso, a hipotensão que pode ser gerada pelo uso de anti-hipertensivos também altera o equilíbrio, predispondo a quedas (Araújo Neto, et al., 2017). A previsão para alguns anos é de que existam ainda mais hipertensos, sendo presumida, por alguns autores, a progressiva redução das metas pressóricas em idosos não frágeis (Andrade, et al., 2017).

Este estudo possui como limitações a amostra selecionada por conveniência e abrangência limitada de um grupo populacional residente em um único bairro da cidade e com características sociodemográficas semelhantes. Além disso, uma outra limitação reside no desconhecimento das causas de tontura dos idosos participantes da pesquisa, tendo em vista que a maioria não possuía um diagnóstico formal que explicasse a ocorrência desse sintoma. Por fim, a última limitação diz respeito ao delineamento transversal, pois este inviabiliza associar a causalidade entre as variáveis estudadas.

\section{Conclusão}

Demonstra-se que a maior parte dos usuários da ESF no município de Serra Talhada, Pernambuco, com déficit de equilíbrio são do sexo feminino, com idade média de 71,1 anos, casados, sedentários e com acúmulo de comorbidades autorrelatadas. Além disso, a amostra de idosos ao apresentar um tempo maior no TUG, comparada aos valores normativos ou de referência, estaria mais predisposta a um evento de queda. Conjuntamente, o estudo concluiu que DM e idade avançada aumentam consideravelmente a chance de dependência e estão, portanto, associados a menor desempenho no teste TUG. A HAS aparenta diminuir a chance de presença de deficiência, sendo necessários outros estudos, com populações distintas, com o objetivo de avaliar essa relação. Assim, através da análise do perfil epidemiológico e da identificação das variáveis que melhor contribuem para a ocorrência probabilística de dependência segundo os critérios do TUG, é possível inferir fatores de risco associados à queda, permitindo uma abordagem rápida e efetiva que poderá diminuir a sua ocorrência, beneficiando os idosos e o sistema de Saúde Pública. Ademais, a partir da análise dos dados obtidos com a pesquisa, espera-se no futuro realizar intervenções com o fito de melhorar a abordagem ao déficit de equilíbrio na população em questão. Espera-se ainda expandir a pesquisa para outras unidades de Estratégia de Saúde da Família, ampliando e diversificando a amostra, bem como realizando um comparativo entre as diferentes populações, levando em conta os fatores sociais atrelados a cada uma delas.

\section{Referências}

Abreu, D. R. D. O. M., Novaes, E. S., Oliveira, R. R. D., Mathias, T. A. D. F., \& Marcon, S. S. (2018). Internação e mortalidade por quedas em idosos no Brasil: análise de tendência. Ciencia \& saude coletiva, 23, 1131-1141.

Acar, S., Demırbüken, İ., Algun, C., Malkoç, M., \& Tekın, N. (2015). Is hypertension a risk factor for poor balance control in elderly adults? Journal of physical therapy science, 27(3), 901-904.

Andrade, I. R., de Almeida Souza, E., da Luz, L. A., \& Júnior, E. P. P. (2017). Características e gastos com hospitalizações por quedas em idosos na Bahia.Ansai, J. H., Andrade, L. P. D., Nakagawa, T. H., \& Rebelatto, J. R. (2018). Performances on the Timed Up and Go Test and subtasks between fallers and non-fallers in older adults with cognitive impairment. Arquivos de neuro-psiquiatria, 76, 381-386.

Araújo, A. H. D., Patrício, A. C. F. D. A., Ferreira, M. A. M., Rodrigues, B. F. L., Santos, T. D. D., Rodrigues, T. D. D. B., \& Silva, R. A. R. D. (2017). Falls in institutionalized older adults: risks, consequences and antecedents. Revista brasileira de enfermagem, 70, 719-725.

Barry, E., Galvin, R., Keogh, C., Horgan, F., \& Fahey, T. (2014). Is the Timed Up and Go test a useful predictor of risk of falls in community dwelling older adults: a systematic review and meta-analysis. BMC geriatrics, 14(1), 1-14. https://doi.org/10.1186/1471-2318-14-14

Battisti I. A. D. \& Smolski F. M. S. (2020). Curso Avançado em Estatística com R da UFFS. smolski.github.io/livroavancado. 
Bischoff, H. A., Stähelin, H. B., Monsch, A. U., Iversen, M. D., Weyh, A., Von Dechend, M., \& Theiler, R. (2003). Identifying a cut-off point for normal mobility: a comparison of the timed 'up and go'test in community-dwelling and institutionalised elderly women. Age and ageing, 32(3), 315-320.

Bohannon, R. W. (2006). Reference values for the timed up and go test: a descriptive meta-analysis. Journal of geriatric physical therapy, 29(2), 64-68. https://doi.org/10.1519/00139143-200608000-00004

Bouccara, D., Rubin, F., Bonfils, P., \& Lisan, Q. (2018). Vertiges et troubles de l'équilibre: démarche diagnostique [Management of vertigo and dizziness]. La Revue de medecine interne, 39(11), 869-874. https://doi.org/10.1016/j.revmed.2018.02.004

Brown, S. J., Handsaker, J. C., Bowling, F. L., Boulton, A. J., \& Reeves, N. D. (2015). Diabetic peripheral neuropathy compromises balance during daily activities. Diabetes care, 38(6), 1116-1122. https://doi.org/10.2337/dc14-1982

Browne, W., \& Nair, B. (2019). The Timed Up and Go test. The Medical journal of Australia, 210(1), 13-14.e1. https://doi.org/10.5694/mja2.12045

Chagas, D. L., Rodrigues, A. L. P., Brito, L. C. \& Soares, E. S. (2018). Relação entre o equilíbrio corporal e o risco de quedas em idosos de um projeto social de Fortaleza-CE. Revista Brasileira de Prescrição e Fisiologia do Exercício, 12(76):547-555.

Ciorba, A., Bianchini, C., Scanelli, G., Pala, M., Zurlo, A., \& Aimoni, C. (2017). The impact of dizziness on quality-of-life in the elderly. European archives of oto-rhino-laryngology: official journal of the European Federation of Oto-Rhino-Laryngological Societies (EUFOS) : affiliated with the German Society for Oto-Rhino-Laryngology - Head and Neck Surgery, 274(3), 1245-1250. https://doi.org/10.1007/s00405-016-4222-z

Coelho Junior, H. J., Rodrigues, B., Aguiar, S. D. S., Goncalves, I. D. O., Pires, F. D. O., Asano, R. Y., \& Uchida, M. C. (2017). Hypertension and functional capacities in community-dwelling older women: a cross-sectional study. Blood pressure, 26(3), 156-165. https://doi.org/10.1080/08037051.2016.1270163

Coelho-Junior, H. J., Rodrigues, B., de Oliveira Gonçalves, I., Asano, R. Y., Uchida, M. C., \& Marzetti, E. (2018). The physical capabilities underlying timed "Up and Go" test are time-dependent in community-dwelling older women. Experimental gerontology, 104, 138-146. https://doi.org/10.1016/j.exger.2018.01.025

Cruz, D. T. D., Ribeiro, L. C., Vieira, M. D. T., Teixeira, M. T. B., Bastos, R. R., \& Leite, I. C. G. (2012). Prevalência de quedas e fatores associados em idosos. Revista de saúde pública, 46, 138-146. https://doi.org/10.1590/S0034-89102011005000087

Cuevas-Trisan, R. (2019). Balance problems and fall risks in the elderly. Clinics in geriatric medicine, 35(2), 173183.https://doi.org/10.1016/j.cger.2019.01.008

Dantas, E. L., de Brito, G. E. G. \& Lobato, I. A. F. (2012). Prevalência de quedas em idosos adscritos à Estratégia de Saúde da Família do município de João Pessoa, Paraíba. Rev APS, 15(1):67-75.

de Freitas Mussi, R. F., Mussi, L. M. P. T., Assunção, E. T. C., \& Nunes, C. P. (2019). Pesquisa Quantitativa e/ou Qualitativa: distanciamentos, aproximações e possibilidades. Revista Sustinere, 7(2), 414-430.

Dobson A.J. (2002). An introduction to generalized linear models. (2a ed.), Boca Raton: Chapman \& Hall.

Doré, A. L., Golightly, Y. M., Mercer, V. S., Shi, X. A., Renner, J. B., Jordan, J. M., \& Nelson, A. E. (2015). Lower-extremity osteoarthritis and the risk of falls in a community-based longitudinal study of adults with and without osteoarthritis. Arthritis care \& research,67(5), 633-639. https://doi.org/10.1002/acr.22499

Dutra M. C., Cabral, A. L. L. \& Carvalho G. A. (2016). Tradução para o português e validação do teste Timed up and go. Rev. Interfaces, Saúde, Hum Tecnol, 3(9):81-88. https://doi.org/X.430.INSS

Ferreira, C. G., Gazzola, J. M., Cendoroglo, M. S., Dias, V. N. \& Ganança, F. F. (2019) Fatores associados ao equilíbrio postural de idosos longevos. Fisioter mov, 32:e003240. https://doi.org/10.1590/1980-5918.032.AO40

Field A. Descobrindo a Estatística usando o SPSS. 2. Artmed; 2011.

Gioacchini, F. M., Albera, R., Re, M., Scarpa, A., Cassandro, C., \& Cassandro, E. (2018). Hyperglycemia and diabetes mellitus are related to vestibular organs dysfunction: truth or suggestion? A literature review. Acta diabetologica, 55(12), 1201-1207. https://doi.org/10.1007/s00592-018-1183-2

Hewston, P., \& Deshpande, N. (2016). Falls and Balance Impairments in Older Adults with Type 2 Diabetes: Thinking Beyond Diabetic Peripheral Neuropathy. Canadian journal of diabetes, 40(1), 6-9. https://doi.org/10.1016/j.jcjd.2015.08.005

Ibrahim, A., Singh, D., \& Shahar, S. (2017). 'Timed Up and Go' test: Age, gender and cognitive impairment stratified normative values of older adults. PloS one, 12(10), e0185641. https://doi.org/10.1371/journal.pone.0185641

Ibrahim, A., Singh, D., Shahar, S., \& Omar, M. A. (2017). Timed up and go test combined with self-rated multifactorial questionnaire on falls risk and sociodemographic factors predicts falls among community-dwelling older adults better than the timed up and go test on its own. Journal of multidisciplinary healthcare, 10, 409-416. https://doi.org/10.2147/JMDH.S142520

Instituto Brasileiro de Geografia e Estatística. Censo 2010 [Internet]. IBGE. https://censo2010.ibge.gov.br/

Jahn K. (2019). The Aging Vestibular System: Dizziness and Imbalance in the Elderly. Advances in oto-rhino-laryngology, 82, 143-149. https://doi.org/10.1159/000490283

Kang, L., Chen, X., Han, P., Ma, Y., Jia, L., Fu, L., Yu, H., Wang, L., Hou, L., Yu, X., An, Z., Wang, X., Li, L., Zhang, Y., Zhao, P., \& Guo, Q. (2018). A Screening Tool Using Five Risk Factors Was Developed for Fall-Risk Prediction in Chinese Community-Dwelling Elderly Individuals. Rejuvenation research, 21(5), 416-422. https://doi.org/10.1089/rej.2017.2005 
Khow, K., \& Visvanathan, R. (2017). Falls in the Aging Population. Clinics in geriatric medicine, 33(3), 357-368. https://doi.org/10.1016/j.cger.2017.03.002

Kojima, G., Masud, T., Kendrick, D., Morris, R., Gawler, S., Treml, J., \& Iliffe, S. (2015). Does the timed up and go test predict future falls among British community-dwelling older people? Prospective cohort study nested within a randomised controlled trial. BMC geriatrics, 15 , 38. https://doi.org/10.1186/s12877-015-0039-7

Kurosawa, C., Shimazu, N., \& Yamamoto, S. (2020). Where do healthy older adults take more time during the Timed Up and Go test? Journal of physical therapy science, 32(10), 663-668. https://doi.org/10.1589/jpts.32.663

Laurence, B. D., \& Michel, L. (2017). The Fall in Older Adults: Physical and Cognitive Problems. Current aging science, 10(3), 185-200. https://doi.org/10.2174/1874609809666160630124552

Long, J., Cai, T., Huang, X., Zhou, Y., Kuang, J., \& Wu, L. (2020). Reference value for the TUGT in healthy older people: A systematic review and metaanalysis. Geriatric Nursing, 41(3), 325-330. https://doi.org/10.1016 / j.gerinurse.2019.11.012

Marchetti, G. F., Whitney, S. L., Redfern, M. S., \& Furman, J. M. (2011). Factors associated with balance confidence in older adults with health conditions affecting the balance and vestibular system. Archives of physical medicine and rehabilitation, 92(11), 1884-1891. https://doi.org/10.1016/j.apmr.2011.06.015

Mari, F. R., Alves, G. G., Aerts, D. R. G. D. C., \& Camara, S. (2016). O processo de envelhecimento e a saúde: o que pensam as pessoas de meia-idade sobre o tema. Revista Brasileira de Geriatria e Gerontologia, 19, 35-44. https://doi.org/10.1590/1809-9823.2016.14122

Mathias, S., Nayak, U. S., \& Isaacs, B. (1986). Balance in elderly patients: the "get-up and go" test. Archives of physical medicine and rehabilitation, 67(6), 387-389.

Moreland, B., Kakara, R., \& Henry, A. (2020). Trends in Nonfatal Falls and Fall-Related Injuries Among Adults Aged $\geq 65$ Years - United States, 20122018. MMWR. Morbidity and mortality weekly report, 69(27), 875-881. https://doi.org/10.15585/mmwr.mm6927a5

National Academies of Sciences, Engineering, and Medicine, Health and Medicine Division, Board on Population Health and Public Health Practice, Committee on Public Health Approaches to Reduce Vision Impairment and Promote Eye Health, Welp, A., Woodbury, R. B., McCoy, M. A., \& Teutsch, S. M. (Eds.). (2016). Making Eye Health a Population Health Imperative: Vision for Tomorrow. National Academies Press (US).

Oliveira, M. R. D., Inokuti, T. T., Bispo, N. N. D. C., Oliveira, D. A. D. A. P., Oliveira, R. F. D., \& Silva, R. A. D. (2015). Elderly individuals with increased risk of falls show postural balance impairment. Fisioterapia em Movimento, 28, 269-276. https://doi.org/10.1590/0103-5150.028.002.AO07

Pavanate, A.A., Hauser, E., Gonçalves, A.K. \& Mazo, G.Z. (2018). Avaliação do equilíbrio corporal em idosas praticantes de atividade física segundo a idade. Rev Bras Ciênc Esporte, 40(4):404-409. https://doi.org/10.1016/j.rbce.2018.03.023

Podsiadlo, D., \& Richardson, S. (1991). The timed "Up \& Go": a test of basic functional mobility for frail elderly persons. Journal of the American Geriatrics Society, 39(2), 142-148. https://doi.org/10.1111/j.1532-5415.1991.tb01616.x

Pondal, M., \& del Ser, T. (2008). Normative data and determinants for the timed "up and go" test in a population-based sample of elderly individuals without gait disturbances. Journal of geriatric physical therapy (2001), 31(2), 57-63. https://doi.org/10.1519/00139143-200831020-00004

Rawlings, J.O., Pantula, S.G. \& Dickey, D.A. (1998). Applied Regression Analysis: a research tools. Springer.

Carbonell, F. R., Ocampo, E. M. H., Pascual, J. M. A., Soler, E., Clapera, F., \& Panicot, J. E. (2014). Experiencia de una Unidad de Prevención de Caídas de un hospital de cuidados intermedios. Revista Española de Geriatría y Gerontología, 49(2), 69-71. https://doi.org/10.1590/1518-8345.2953-3145

Rodrigues, A., Assef, J. C., \& Lima, C. B. (2019). Assessment of risk factors associated with falls among the elderly in a municipality in the state of Paraíba, Brazil. A cross-sectional study. Sao Paulo medical journal = Revista paulista de medicina, 137(5), 430-437. https://doi.org/10.1590/15163180.2018.0198120619

Rodrigues, M. M. P., Falcão, R. M. D. M., Veras, R. F. S., Barbosa, K. T. F., de Oliveira, F. M. R. L., Pereira, M. A., ... \& dos Santos Oliveira, J. (2017). Timed up and go risk predictor of falls in elderly people residing in the community? International archives of medicine, 10.

Roshdibenam, V., Jogerst, G. J., Butler, N. R., \& Baek, S. (2021). Machine Learning Prediction of Fall Risk in Older Adults Using Timed Up and Go Test Kinematics. Sensors (Basel, Switzerland), 21(10), 3481. https://doi.org/10.3390/s21103481

Rubenstein, L. Z., \& Josephson, K. R. (2006). Falls and their prevention in elderly people: what does the evidence show?. The Medical clinics of North America, 90(5), 807-824. https://doi.org/10.1016/j.mcna.2006.05.013

Santos, R. K. M., Maciel, A. C. C., Britto, H. M. J. S., Lima, J. C. C. \& Souza T. O. (2015). Prevalência e fatores associados ao risco de quedas em idosos adscritos a uma Unidade Básica de Saúde do município de Natal, RN, Brasil. Cien Saude Colet, 20(12):3753-3762. https://doi.org/10.1590/1413812320152012.00662015

Schlick, C., Schniepp, R., Loidl, V., Wuehr, M., Hesselbarth, K., \& Jahn, K. (2016). Falls and fear of falling in vertigo and balance disorders: A controlled cross-sectional study. Journal of vestibular research: equilibrium \& orientation, 25(5-6), 241-251. https://doi.org/10.3233/VES-150564

Silva, E. M. T. D., Lima, B. F. D., Mantello, É. B., Sousa, A. G. P. D., Diniz, J., \& Gazzola, J. M. (2019). Diseases and symptoms associated with changes in postural balance in diabetics: an integrating literature review. Revista CEFAC, 21. https://doi.org/10.1590/1982-0216/201921617318

Sorond, F. A., Cruz-Almeida, Y., Clark, D. J., Viswanathan, A., Scherzer, C. R., De Jager, P., Csiszar, A., Laurienti, P. J., Hausdorff, J. M., Chen, W. G., Ferrucci, L., Rosano, C., Studenski, S. A., Black, S. E., \& Lipsitz, L. A. (2015). Aging, the Central Nervous System, and Mobility in Older Adults: Neural Mechanisms of Mobility Impairment. The journals of gerontology. Series A, Biological sciences and medical sciences, 70(12), 1526-1532. https://doi.org/10.1093/gerona/glv130 
Research, Society and Development, v. 10, n. 12, e262101220277, 2021

(CC BY 4.0) | ISSN 2525-3409 | DOI: http://dx.doi.org/10.33448/rsd-v10i12.20277

Stolt, L. R. O. G., Kolisch, D. V., Tanaka, C. \& Cardoso, M. R. A. (2020), Schmitt ACB. Increase in fall-related hospitalization, mortality, and lethality among older adults in Brazil. Rev Saude Publica, 54:76. https://doi.org/10.11606/s1518-8787.2020054001691

Suzman, R., Beard, J. R., Boerma, T., \& Chatterji, S. (2015). Health in an ageing world--what do we know? Lancet (London, England), 385(9967), 484-486. https://doi.org/10.1016/S0140-6736(14)61597-X2.

Tavares, R. E., Jesus, M. C. P. D., Machado, D. R., Braga, V. A. S., Tocantins, F. R., \& Merighi, M. A. B. (2017). Envelhecimento saudável na perspectiva de idosos: uma revisão integrativa. Revista brasileira de geriatria e gerontologia, 20, 878-889. https://doi.org/10.1590/1981-22562017020.170091

Timar, B., Timar, R., Gaiță, L., Oancea, C., Levai, C., \& Lungeanu, D. (2016). The Impact of Diabetic Neuropathy on Balance and on the Risk of Falls in Patients with Type 2 Diabetes Mellitus: A Cross-Sectional Study. PloS one, 11(4), e0154654. https://doi.org/10.1371/journal.pone.0154654

Vanspauwen R. (2018). Tontura e medo de cair em idosos: alguns fatos. The journal of international advanced otology, 14(1), 12. https://doi.org/10.5152/iao.2018.0201815

Vennu, V., \& Bindawas, S. M. (2014). Relationship between falls, knee osteoarthritis, and health-related quality of life: data from the Osteoarthritis Initiative study. Clinical interventions in aging, 9, 793-800. https://doi.org/10.2147/CIA.S62207

Waisman G. (2017). Hipertensión arterial en el anciano [Arterial hypertension in the elderly]. Hipertension y riesgo vascular,34(2), 61-64. https://doi.org/10.1016/j.hipert.2017.03.004

Zalewski C. K. (2015). Aging of the Human Vestibular System. Seminars in hearing, 36(3), 175-196. https://doi.org/10.1055/s-0035-1555120 